\title{
LOS LÍMITES DE LA RACIONALIDAD CIENTÍFICA FRENTE AL FENÓMENO DE LA ESPIRITUALIDAD: UNA REVISIÓN EPISTEMOLÓGICA DE LOS REDUCCIONISMOS METODOLÓGICOS EN LA INVESTIGACIÓN PSICOCLÍNICA
}

\section{THE LIMITS OF SCIENTIFIC RATIONALITY AGAINST THE PHENOMENON OF SPIRITUALITY: \\ AN EPISTEMOLOGICAL REVIEW OF METHODOLOGICAL REDUCTIONISM IN PSYCHO-CLINICAL RESEARCH}

\section{María Teresa Gargiulo ${ }^{a^{*}}$}

Fechas de recepción y aceptación: 2 de septiembre de 2019 y 29 de abril de 2020

Resumen: El artículo ofrece un análisis exploratorio respecto a los límites y obstáculos que presentan los reduccionismos metodológicos en la investigación psicoclínica respecto a la espiritualidad. Por reduccionismo metodológico se entiende el proceso de simplificación y operatividad que exige el mismo diseño experimental adoptado en la investigación psicoclínica. El trabajo se limita a señalar un absurdo que se sigue de la misma implementación de ciertos recortes metodológicos: a saber, aquel diseño metodológico que debería garantizar el abordaje racional de los fenómenos espirituales y religiosos opera muchas veces como facilitador de comprensiones erróneas e incompletas del fenómeno. En este sentido quizás podamos repensar estos diseños de la investigación psicoclínica incorporando o atendiendo en sus formulaciones teóricas al bagaje cognoscitivo de las tradiciones teológicas y espirituales -elementos y principios aparentemente ajenos a la racionalidad

${ }^{a}$ Facultad de Filosofía y Letras. Universidad Nacional de Cuyo. Investigadora del CONICET (Consejo Nacional de Investigaciones Científicas y Técnicas de Argentina).

* Correspondencia: Centro Universitario, M5502JMA. Facultad de Filosofía y Letras, Universidad Nacional de Cuyo. Mendoza. Argentina.

E-mail: gargiulomteresa@gmail.com. 
científica-. Pues ellas pueden significar un camino posible para que las demostraciones científicas de la salud mental adquieran mayor fuerza explicativa.

Palabras clave: reduccionismo, investigación psicoclínica, epistemología de la psicología, validación empírica, racionalidad científica.

Abstract: The article offers an exploratory analysis regarding the limits and obstacles presented by methodological reductionisms in psycho-clinical research regarding spirituality. Methodological reductionism means the process of simplification and operability required by the same experimental design adopted in psycho-clinical research. The work is limited to pointing out an absurdity that follows from the same implementation of certain methodological cuts: that is, that methodological design that should guarantee the rational approach to spiritual and religious phenomena often operates as a facilitator of erroneous and incomplete understandings of the phenomenon. In this sense, perhaps we can rethink these designs of psycho-clinical research by incorporating or assisting in their theoretical formulations the cognitive baggage of theological and spiritual traditions -elements and principles apparently unrelated to scientific rationality-. Since they can mean a possible way for scientific demonstrations of mental health to acquire greater explanatory power.

Keywords: spirituality, psycho-clinical research, reductionism, psychotherapeutic efficacy, psychology of religion.

\section{INTRODUCCIÓN}

Nuestro estudio tiene la intención de ser parte del esfuerzo mancomunado que existe en nuestros días por dilucidar el alcance y los límites epistémicos de los reduccionismos metodológicos adoptados en la investigación psicoclínica. El botón de muestra que nos permitirá ilustrar la operatividad de tales reduccionismos será un conjunto de estudios relativamente recientes que buscan medir la eficacia de ciertas prácticas espirituales en la psicoterapia.

La pregunta que abre nuestro estudio inquiere acerca del alcance y peso que poseen estos estudios de tipo empírico-experimental. ¿Dichos análisis 
pueden erigirse como materia rectora y orientadora de la práctica psicoterapéutica? ¿Miden efectivamente el fenómeno que reconocemos bajo el nombre de espiritualidad?

En línea, con el esfuerzo por dilucidar cuestiones nodales sobre metodología de la investigación científica aplicada (clínica) cabe destacar los trabajos de Kazdin (2001, 2008), Barlow (1981), Roberts y Ilardy (2003), Finger y Rand (2003) y el de Anderson y Kim (2003), quienes analizan, entre otros, las amenazas de validación de los diseños de investigación y se esfuerzan por formular diseños controlados para evaluar la eficacia de la intervención psicoterapéutica.

Por otro lado, disponemos de una serie de estudios que revelan particularmente los reduccionismos que operan en las diversas modalidades de estudios de las ciencias de la salud mental. Se pueden consultar los estudios de Murphy (2005; 1998), Zizioulas (2006), Taylor (2007), Cooper (2007), Barbour (1997), Barrett, Dunbar y Lycett (2002), Ellis (2002), Bennett y Hacker (2003), Faucher (2006), Kim (2006) y Peacocke (1993; 2002) sobre los reduccionismos de tipo ontológico. Respecto a los reduccionismos epistémicos y metodológicos se pueden ver los trabajos de Smith (2000), Andler (2006). Kistler (2006), Marras (2006), Poirier (2006), Schaffner (2006), Vande Kemp (2000), Watts (2002), Gallego et al. (2016).

En continuidad con esta tradición de trabajo, nuestro estudio se circunscribe a explorar una serie de paradojas o absurdos que se siguen de la implementación de ciertos reduccionismos metodológicos en la investigación psicoclínica. En este sentido, el valor del presente trabajo no radica tanto en la originalidad o novedad de su tesis sino, más bien, en la sistematización o precisión con la que busca identificar una particular paradoja: el diseño experimental de investigación que deberían garantizar, en este caso, el abordaje racional de las realidades espirituales y religiosas, al implementar ciertos reduccionismos, opera muchas veces como facilitador de comprensiones erróneas e incompletas de tales fenómenos.

En orden a ser autoconsistentes en nuestro planteo, presentaremos, los reduccionismos metodológicos básicos de los diseños experimentales psicoclínicos y señalaremos cómo estos pueden comportarse como amenazas de validación de los mismos diseños. Estructuraremos nuestro trabajo en cuatro 
partes. Primero, haremos una breve reseña histórica de la implementación de los reduccionismos metodológicos en la investigación psicoclínica (1). Luego, pasaremos a analizar aquellos recortes que atentan contra la validez interna del diseño de investigación (2). A continuación, expondremos aquellas simplificaciones metodológicas que constituyen una amenaza contra la validez del constructo (3). Finalmente, señalaremos la invalidez externa que parece seguirse de la adopción de determinados reduccionismos metodológicos en la investigación psicoclínica (4).

\section{$\S 1$. BREVE RESEÑA DE LA INVESTIGACIÓN PSICOCLÍNICA}

Para poder delinear el objeto de nuestro estudio es necesario distinguir la psicopraxiología clínica de la investigación psicoclínica. La primera ha sido definida por la APA como "como cualquier servicio psicológico provisto por un profesional entrenado que usa primordialmente formas de comunicación e interacción para evaluar, diagnosticar y tratar reacciones emocionales, formas de pensamiento y patrones conductuales disfuncionales de un individuo, familia o grupo" (American Psychological Association, 2010: 474).

La investigación clínica, en cambio, exige la producción metódica y controlada de conocimiento sobre cuestiones clínicas, como lo son la naturaleza de los trastornos psicológicos, la eficiencia y efectividad de las diversas prácticas psicoterapéuticas. Pues bien, nuestro interés está puesto no en la tarea psicoterapéutica sino más bien en la investigación psicoclínica que se desarrolla a través de diseños experimentales; particularmente aquella que se ordena a probar la eficiencia terapéutica de ciertas prácticas espirituales.

En los últimos años ha crecido exponencialmente el cuerpo de literatura que presenta la espiritualidad como un área importante no solo de la evaluación diagnóstica, sino incluso de la misma instancia terapéutica. Probablemente el resurgimiento del interés por esta temática se explique a propósito de la inclusión en el DSM-IV (2000) de la categoría "Problema Religioso o Espiritual” Z71.7 (V62.89). Esta nueva categoría buscaría contemplar y atender a las dificultades o malestar psíquico que puede suponer la pérdida o el cuestionamiento de la fe, los problemas asociados con la conversión a una nueva fe, 
o el cuestionamiento de los valores espirituales. Dicha incorporación parece recoger una larga discusión acerca de si la espiritualidad es un área importante que desata o paraliza dinamismos psíquicos que son relevantes para el desarrollo de la personalidad. ${ }^{1}$ Dentro de una amplia variedad de posiciones hoy parece estar fuera de discusión que un determinado tipo de espiritualidad pueda ser causa de depresión, manía, masoquismo, histeria, fobias o comportamiento obsesivo. $\mathrm{O}$, por el contrario, también puede suceder que algunos valores o prácticas religiosas se traduzcan en hábitos o estructuras psíquicas saludables. En este último caso, la religión y la vida espiritual representarían factores protectores y promotores de la salud mental.

Pues bien, para responder a la pregunta acerca de la eficacia terapéutica de las prácticas espirituales -así como de los efectos de cualquier forma o práctica de psicoterapéutica respecto a un trastorno particular, la psicología ha tomado prestado de la medicina sus diseños de investigación-. Adopta diseños metodológicos que originalmente fueron formulados y aprobados por la Administración de Alimentos y Medicamentos (FDA) de Estados Unidos. Nos referimos particularmente al diseño aleatorio que fue desarrollado entre 1920 y 1930; y el diseño de grupo de control con placebo desarrollado en la década de 1950.

El énfasis que en la década de los noventa se dio a la necesidad de estandarizar los tratamientos médicos y de proporcionar evidencia respecto a la eficacia de cada tratamiento específico -incluso aquellos que estaban dirigidos a los trastornos mentales- llevó a un grupo de trabajo de la División 12 de la Asociación Americana de Psicología (APA) a trabajar en esta misma línea metodológica: "Si la psicología clínica ha de sobrevivir en este apogeo de la psiquiatría biológica, la APA debe actuar para enfatizar la fuerza de lo que somos" (...) "tienen que ofrecer una variedad de psicoterapias de eficacia probada" (Task Force on Promotion and Dissemination of Psychological Procedures, 1995: 3).

${ }^{1}$ La espiritualidad no solo es entendida como un aspecto de la personalidad, sino que ella en sí misma operaría como una función integradora de la personalidad (véase Becker, 2001; MacInnes, 2003: 51; Ingersoll, 1994; Roof, 1999: 35). Ella configuraría la unidad interna, la autopercepción y las modalidades de las relaciones interpersonales (Schneiders, 1998; McGrath, 2006; Kosek, 1996; Theophan, 1995, pp. 95-99; Wagener y Malony, 2006; Wuthnow, 1998; Wiseman, 2007). 
La adopción de tales esquemas metodológicos de investigación por parte de la APA exigía remplazar la explicación biológica por una explicación psicológica, es decir, discernir en el grupo experimental mecanismos de cambio y acciones terapéuticas de naturaleza psicológica. Un tratamiento psicoterapéutico específico estaría validado empíricamente siempre que al menos dos estudios demuestren la superioridad de una acción terapéutica sobre un grupo experimental con un trastorno o problema específico frente a los grupos de control o tratamientos alternativos (tratados con placebo).

No obstante, al mismo tiempo que estos diseños eran implementados en la investigación psicoclínica, la misma APA indicaba la necesidad de advertir a los psicoterapeutas las limitaciones y recortes que suponían tales diseños: "la práctica basada en la evidencia requiere que los psicólogos reconozcan las fortalezas y limitaciones de la evidencia obtenida de diferentes tipos de investigación" (APA Presidential Task Force on Evidence-Based Practice, 2006: 275). En línea con esta advertencia, creemos necesario discernir puntualmente los recortes metodológicos de aquellos diseños experimentales que procuran medir la eficacia psicoterapéutica de ciertas prácticas espirituales.

Por reduccionismo entendemos el proceso de simplificación y operatividad que exige el mismo diseño experimental adoptado por la investigación psicoclínica. Si bien el reduccionismo que se implementa en un diseño experimental goza de toda una suerte de ventajas, también tiene sus peligros. Podría decirse que existe una compensación, a saber, las explicaciones reduccionistas ganan en simplicidad mientras pierden en precisión. El diseño experimental exige la selección de un conjunto parcial de variables y descartar otros factores o aspectos importantes. Requiere, habitualmente, eliminar cosas que necesitan ser entendidas y que son parte de una imagen completa del fenómeno bajo estudio. En este sentido, dichos diseños de investigación pueden ofrecer explicaciones excesivamente incompletas e incluso erróneas respecto al fenómeno que pretenden medir. Pues bien, son estos mismos recortes o simplificaciones - como veremos a continuación- los que pueden atentar contra la misma validez de la investigación psicoclínica. 


\section{§2. LA SELECCIÓN DE VARIABLES O LA AMENAZA DE LA VALIDEZ INTERNA}

Los diseños experimentales requieren establecer una definición operacional del problema o trastorno a intervenir. Cabe aclarar que lo psíquico, y mucho menos lo espiritual, no es medible pues no se trata de realidades extensas y cuantificables. De aquí que estos diseños busquen medir las dinámicas psíquicas concomitantes a las prácticas espirituales a través de sus cualidades, de sus efectos o del resultado de sus operaciones. Se trata de traducir dichas dinámicas a sus exteriorizaciones fenoménicas de modo tal que ellas constituyan ciertas formas de percepción y posibilidades de comprobación que procedan del propio objeto. Esto es, traducir a indicadores observables o referentes empíricos como actos de habla o conductas que manifiesten específicamente el problema a estudiar.

Las manifestaciones fisiológicas, conductuales, lingüísticas, cognitivas o interpersonales de la vida espiritual permiten establecer una línea de base que no es sino el parámetro comparativo que permite evaluar sus posibles efectos psicoterapéuticos ${ }^{2}$.

Entre los estudios de tipo empírico que se publicaron en las bases de datos de MEDLINE y PsychINFO entre los años 1996-2007, Vachon, Fillion y Achille (2009) discriminan 11 dimensiones a las que se hace referencia bajo el término espiritualidad: 1) el propósito que le da sentido a la vida; 2) la auto-trascendencia que permite estar en armonía y en paz consigo mismo; 3) la trascendencia hacia un ser superior; 4) un sentimiento de comunión con el propio yo, con Dios, con el Universo o la Naturaleza dentro de una red de relaciones interpersonales; 5) un cuerpo de creencias que son objeto de fe;

${ }^{2}$ La determinación de una línea de base posibilita, en definitiva, no solo conocer el tenor fáctico de las alteraciones que afectan al paciente al inicio del estudio, sino sobre todo poder predecir si los cambios registrados en él se deben efectivamente a la terapia o si se deben a una remisión espontánea, a factores inespecíficos, o a la propia maduración del paciente etc. Anderson \& Kim (2003: 78) y Kazdin (2001) desarrollaron un diseño denominado ABAB, que permite dirimir con cierta evidencia si los cambios producidos desde la línea de base se deben a la intervención psicoterapéutica original o son efecto de una variable extraña. Para ello, una vez que se han obtenido cambios desde la línea de base, se retorna a una fase sin tratamiento. De ese modo, si el paciente en esa fase remite a tasas de respuesta semejantes a las de la primera fase, es posible predecir que tales cambios de mejoría se debieron al tratamiento. Finalmente este diseño permite replicación pues al reinstaurar la intervención nuevamente es posible discernir si los cambios se deben o no al tratamiento original.

SCIO. Revista de Filosofía, n. ${ }^{\circ}$ 18, Julio de 2020, 261-294, ISSN: 1887-9853 
6) la esperanza entendida como una actitud frente a las dificultades de la vida; 7) la actitud frente a la muerte; 8) el aprecio a la vida; 9) los valores personales; 10) un proceso dinámico y 11) consciente.

Atendiendo a esta variopinta referencia teórica que posee la noción de espiritualidad en la literatura existente, los diseños experimentales la traducen en múltiples y diversas variables específicas en orden a poder medir cómo estas se relacionan con otra variable como puede ser la reducción de estrés, o cualquier otra variable relacionada con la salud mental. A saber, no es la espiritualidad, en toda su complejidad y dinámica, la que cae bajo la consideración del diseño. Por el contrario, en los diseños experimentales se seleccionan variables o indicadores observables, evitando constructos abstractos o genéricos. Se la traduce en términos de la asistencia al servicio de adoración, el desempeño de prácticas religiosas como la oración, o en términos de los vínculos interpersonales que se establecen en las comunidades religiosas, etc. El carácter polifacético de la espiritualidad es reducido a una o dos variables de este tipo.

Se trata de análisis funcionales donde la religión y la espiritualidad son medidas en los términos de sus funciones para promover o deteriorar la salud física y mental, por ejemplo, en términos de los recursos que provee para lidiar con el estrés de la vida.

Disponemos de estudios que sostienen que la participación en ritos y comunidades religiosas parece proteger a las personas contra las enfermedades y promover la salud. Un grupo de investigaciones muestra la correlación que existe entre la frecuente asistencia a una iglesia y la reducción de las tasas de mortalidad: hay un aumento en la vida útil de las personas de hasta 7 años, a saber, un beneficio para la salud equivalente al hecho de no fumar (Levin, Chatters y Taylor, 2005; Ellison y Levin, 1998; Hummer, Rogers, Nan y Ellison, 1999; Koenig, Hays et al., 1999; McCullough, 2001; McCullough, Hoyt, Larson, Koenig y Thoresen, 2000; Koenig, 2001; Thoresen, Harris y Omán, 2001; Mueller, Plevak y Rummans, 2001; Shahabi et al., 2002; Contrada et al., 2004; Oxman, Freeman, y Manheimer, 1995; Chatters, 2000; Levin y Chatters, 1998). La práctica religiosa también se asocia con tasas más bajas de depresión y de enfermedades cardiovasculares debido a que la asistencia a comunidades religiosas aparentemente reduciría la presión arterial y la hi- 
pertensión (Koenig, 1995; Krause, 2002; Steffen, Hinderliter, Blumenthal y Sherwood, 2001; Koenig, Cohen, Blazer, Pieper et al., 1992). Esta misma variable también está correlacionada con menores índices de accidentes cerebrovasculares, de enfermedades pulmonares, y de deterioro cognitivo (Maselko, Kubzansky, Kawachi, Staudenmayer y Berkman, 2006; Van Ness y Kasl, 2003). La asistencia a prácticas religiosas parece estar relacionada con mejoras a largo plazo en la discapacidad y el manejo del dolor crónico, la búsqueda de fuentes de fortaleza y el descubrimiento de significado en el sufrimiento (Idler, 1995; Idler y Kasl, 1997a, 1997b; Cohen y Pressman, 2006; Kirby, Coleman \& Daley, 2004).

Ahora bien, el recorte fenomenológico de la espiritualidad, su traducción a una variable cuantificable -como puede ser la participación o asistencia a prácticas religiosas- no puede constituir una variable específica debido a la ambigüedad o múltiple significación que puede tener en una población este patrón de comportamiento. Y aun admitiendo que tal patrón refleja al menos parcialmente la espiritualidad de la población analizada, nada dice acerca de las características del compromiso, de la madurez, de la satisfacción espiritual o de las luchas religiosas que puede estar atravesando el grupo experimental. En pocas palabras, nada dice acerca de si esta dimensión de la personalidad opera en la población estudiada como un auténtico factor protector de la salud o si esta se debe a otras variables no consideradas en el estudio. Luego, la participación en comunidades religiosas no puede comportarse en el diseño como una variable específica con una incidencia unilateral sobre la salud física o mental.

La selección de una variable ambigua atenta contra la misma validez interna de la investigación. Se dice que hay validez interna cuando los resultados de la investigación pueden atribuirse a una variable específica. El problema aquí, entiéndase bien, es si realmente la espiritualidad está verdaderamente representada en la variable seleccionada y, por tanto, si está relacionada efectivamente con los resultados que arrojan los estudios. Dicho en otros términos: bajo una supuesta variable específica se reúne una amplia variedad de comportamientos. Sin realizar prueba alguna de homogeneidad se reconocen las "manzanas y naranjas" como una misma variable, desconociendo las amplias diferencias que existen entre ambas. ¿Acaso los efectos producidos por 
las manzanas no son diferentes a los efectos producidos por las naranjas? Es necesario identificar diversas modalidades de pertenencia o participación en comunidades religiosas de modo que la variabilidad de los resultados refleje estas diferencias.

En una situación análoga se encuentran los estudios relativos a la aparente eficiencia psicoterapéutica de la oración, a saber, un patrón de comportamiento que refiere a una realidad tan multidimensional como ambigua (Masters, 2005; Brown, 1994; Krause, Chatters, Meltzer y Morgan, 2000; Finney y Malony, 2001). Según sea la tradición religiosa el término oración refiere a prácticas absolutamente diversas, y por ello la estructura cognitiva y las dinámicas emocionales concomitantes a dicha práctica pueden resultar inconmensurables. Aun cuando se circunscriba la práctica de la oración a una tradición religiosa particular como puede ser la cristiana, se reconocen en ella diversos tipos de oración: la meditación (la simple escucha, la adoración, la experimentación de un tipo de presencia); la oración ritual, la oración petitoria, la oración coloquial ${ }^{3}$.

En pocas palabras, los citados análisis funcionales pasan por alto las diferencias sustantivas que pueden co-existir entre los miembros de una población acerca de su manera de comprender y vivenciar las variables seleccionadas. Ciertamente el carácter genérico y ambiguo de estas variables da cuenta de la dificultad que suponen ciertos recortes metodológicos requeridos en los estudios clínicos. Quizás porque la realidad de la espiritualidad y las experiencias religiosas de una población son lo suficientemente complejas como para ser asidas por los citados estudios, sea por la combinación de variables que dichas dinámicas suponen, sea por la forma individual que ellas operan en cada paciente (Thoresen et al., 2001). Hasta aquí podríamos enunciar al menos la necesidad de que tales variables recogieran el contexto teológico, cultural, vivencial y relacional de la espiritualidad y la religión.

\footnotetext{
${ }^{3}$ En este sentido cabe destacar el estudio de Laird, Snyder, Rapoff y Green (2004), quienes formularon un inventario de oración multidimensional para distinguir entre la oración centrada en el problema que involucra la súplica o la confesión y la oración centrada en la emoción como la adoración y la acción de gracias. Banziger, van Uden y Janssen (2008), por su parte, postulan una correlación entre los diferentes tipos religiosos de oración y los estilos de afrontamiento colaborativos o diferidos.
} 
Existe también otro tipo o modelo de estudio que evalúa los problemas de naturaleza espiritual en base a herramientas o cuestionarios específicos. Se trata de diversas diseños de investigación que implementan herramientas de evaluación espiritual tales Protocolo Rush para detectar la lucha religiosaespiritual (Fitchett y Risk, 2009); la herramienta de evaluación de un solo ítem de Steinhauser que se consigna bajo la pregunta "¿Está usted en paz?" (Steinhauser, Clipp y Bosworth, 2004; Steinhauser, 2006); el cuestionario WHOQOL-SRPB (WHOQOL Group, 1998); la herramienta de detección de Mako, que inquiere fundamentalmente si el paciente tiene dolor espiritual (Mako, Galek y Poppito, 2006); o la escala de lesiones espirituales (Fitchett et al., 1999). A partir de tales cuestionarios se selecciona un grupo particular de variables, definidas no ya en los términos de asistencia a una comunidad o por ciertas prácticas religiosas, sino por instancias más genéricas tales como el sentido de la vida, la relación con la transcendencia, la capacidad de entender a otros, etc.

Aunque tales estudios significan ciertamente un progreso en la tarea de estandarizar las evaluaciones del problema espiritual, estos tienden a profundizar la brecha entre la significación religiosa y los métodos de detección del problema espiritual. Presentan un problema análogo a los diseños experimentales citados más arriba. Se trata de investigaciones que no proporcionan dato alguno respecto al contenido y la evolución en la que se padece un problema espiritual tal como ha sido apuntado por Gallego et al. (2016) y por Balboni et al. (2017). Nuevamente: la selección de variables no puede hacerse a costa de perder el conocimiento que proveen las tradiciones religiosas. Pues estas permiten, de una u otra manera, dar cuenta de las estructuras cognitivo-emocionales que intervienen en una experiencia espiritual.

En última instancia, la ambigüedad de tales variables acusa quizás los excesos de la radical distinción que la literatura ha establecido entre espiritualidad y religión.

Prácticamente existe un consenso respecto a la distinción entre espiritualidad y religión. Mientras que a la religión se la define como un sistema de creencias, rituales y cultos de una institución o comunidad organizada en vistas a establecer una relación con un ser divino (Sinclair, Pereira y Raffin, 2006); la espiritualidad es entendida como una realidad más inclusiva. Ella 
es asociada a experiencias subjetivas con fuerte significación personal (Rumbold, 2003).

Ciertamente la investigación psicoclínica está interesada en analizar los mecanismos psicológicos subyacentes de las vivencias subjetivas y dinámicas de una población, y no en los aspectos institucionales y doctrinarios de la religión. Sin embargo, la exacerbación de esta escisión entre espiritualidad y religión podrían dejar a la investigación clínica con un concepto oscuro y vacío de espiritualidad. Pues apelando a los principios teóricos y semánticos de una tradición religiosa es como la investigación podría acceder a las estructuras valorativas, emotivas y cognitivas de las experiencias de trascendencia de una población. ¿Cómo podrán develarse las estructuras cognitivas y emocionales de una particular experiencia espiritual sin comprender el marco semántico que una población le asigna a tal experiencia? Son las tradiciones religiosas las que han retratado múltiples formas y modalidades de vida espiritual y en este sentido son ellas las que permitirían interpretar y hacer comprensible la experiencia espiritual de una población seleccionada.

De un modo u otro, la situación descrita pone en jaque la exacerbada escisión que las investigaciones psicoclínicas trazan entre la base fenoménica susceptible de ser abordada empíricamente y el cuerpo semántico que es recluido a una instancia pseudo-científica. Pues ante la tarea de definir y seleccionar variables específicas que efectivamente sean representativas de un particular tipo de espiritualidad, es necesario recoger y revisar el bagaje de las tradiciones religiosas.

\section{§3. Modelos y MeCANismos DE ACCión o LA AMENAZA DE VAlideZ DEL CONSTRUCTO}

Otro de los requisitos básicos de estos diseños experimentales es tratar de identificar los mecanismos psicológicos específicos que intervienen en las prácticas religiosas y su incidencia en la salud mental. Es necesario -explican Baker et al. (2008)- demostrar evidencia formal respecto a sus mecanismos de acción específicos. Pues la ausencia de tales mecanismos deja abierta 
la posibilidad de que la intervención pueda simplemente capitalizar rituales inespecíficos, no creíbles o efectos placebo (Baker et al., 2008: 72).

Las deficiencias en la formulación de esta explicación teórica son reconocidas en la literatura como la amenaza de validez del constructo (Finger y Rand, 2003: 22; Kazdin 2001: 16). Se trata de la incapacidad del constructo o base conceptual para dar cuenta de la dinámica propia de la variable estudiada. Son investigaciones psicoclínicas que se desarrollan en un marco de orfandad conceptual respecto a los parámetros desde los cuales es posible calibrar el grado de validez de los datos obtenidos en la investigación. ¿Por qué la práctica religiosa tiene un efecto en el comportamiento bajo examen? ¿Por qué la frecuente asistencia a un rito religioso se traduce en una mayor tasa de supervivencia o en un menor índice de un grupo específico de enfermedades?

Entre las investigaciones que adolecen de una justificación teórica limitada podemos citar una serie de estudios que pretenden mostrar una aparente correlación entre la oración de intercesión y las mejoras en el curso de los tratamientos hospitalarios (Byrd, 2001; Harris et al., 2001); o entre la práctica de la oración y la disminución de la depresión y la angustia después de la cirugía de derivación coronaria (Ai, Dunkle, Peterson y Bowlling, 1998). Se trata de estudios que suponen prácticamente una noción mágica o mecánica de la oración de intercesión por la cual se espera, como efecto medible, que aquella cure específicamente la enfermedad que se demanda. No habría, aparentemente, otros mecanismos psicológicos o fisicoquímicos que dieran cuenta del efecto curativo de la oración, que una acción divina o mágica. Esto ha sido denunciado de una u otra manera por Levin (1996, 2001: 102), Dossey (1997), Miller (2001) y Francis y Evans (2001).

Este diseño de explicación teórica estaría invalidado no solo por la racionalidad científica, en cuanto que se configura como una hipótesis ad hoc que da por demostrado lo que en realidad deberían demostrar, sino también por ciertas tradiciones religiosas, particularmente la cristiana en cuanto que le es ajena esta comprensión mágica y mecánica de la oración.

En otro amplio grupo de estudios prima lo que es conocido como el modelo de amortiguamiento, el cual predice que la religión resulta útil en la medida que los factores de estrés tienen menos impacto en los participantes activos. Las prácticas religiosas generarían procesos o dinámicas de relajación (Dillon 
y Wink, 2003, 2007: 138 y 186-193; Wink, 2003; Kennedy y Drebing, 2002; Krause y Tran, 1989; Levin y Chatters, 1998; Koenig, 2001; McCullough, Pargament, y Thoresen, 2000; Kim et al., 2005; George et al., 2002; Levin, 2001: 76; Arcury, Quandt, McDonald y Bell, 2000). Dentro de este modelo de amortiguamiento existen tres modalidades específicas para explicar la relación entre religión y salud: el modelo moderador, el supresor y el contrabalanceado (Krause y Tran, 1989; Chatters, 2000).

El modelo contrabalanceado es un tipo de modelo de mediación, que sostiene que los efectos de la religión en la salud ocurren indirectamente a través de una variable intermedia. Por ejemplo, la actividad religiosa puede mejorar las habilidades de afrontamiento que actúan para disuadir la angustia o mejorar la salud (McCullough y Smith, 2003; Davidson, Pizzagalli, Nitschke y Putnam, 2002; Baetz, Griffin, Bowen, Koenig y Marcoux, 2004; McCullough y Larson, 1999; Kendler, Gardner et al., 1997; Bosworth, Park, McQuoid, Hays y Steffens, 2003).

Los modelos moderador y supresor sostienen que la participación religiosa tiene un efecto directo y positivo en la salud, pero difieren entre sí en su comprensión acerca de cómo este proceso puede funcionar. En el moderador, la religión tiene un mayor impacto cuando los niveles de estrés son altos, aunque el estrés no afecta el nivel real de la práctica religiosa. ${ }^{4} \mathrm{El}$ modelo supresor dicta que a un mayor índice de problemas se desencadenan mayores niveles de práctica religiosa, la cual suprime los efectos del estrés (Pargament, 1997: 306; Levin y Chatters, 1998).

Respecto a las variables intermedias que podrían explicar cómo la religión puede tener un efecto beneficioso en la salud, se discriminan diversos mecanismos de acción:

Unos estudios apuntan que en tanto las religiones como las diversas modalidades de espiritualidad promueven un estilo de vida saludable y desalientan los hábitos destructivos como fumar o el consumo excesivo de alcohol,

\footnotetext{
${ }^{4}$ Flannelly, Koenig, Ellison, Galek y Krause (2006) alegan que la religión parece actuar proporcionando un efecto amortiguador tanto más efectivo cuanto mayor sea el estrés o el riesgo de depresión que afecta a una población.
} 
fomentan el cuidado de la salud mental (Peltzer, 2004; Koenig, Hays et al., 1999; Aaron, Levine y Burstin, 2003).

Otras investigaciones postulan que es el contenido específico de las creencias religiosas, lo que brinda seguridad existencial y permite desarrollar habilidades de afrontamiento. Entre tales creencias se citan (a) las percepciones positivas de uno mismo y de los otros; (b) una cosmovisión que promueve la esperanza y la reducción de la incertidumbre; (c) un sentido de la cercanía y protección de Dios; (d) la creencia de que todos los eventos son parte de un plan; (e) las ideas sobre una buena vida futura (Krause et al., 2002; Chatters, 2000; Musick, Traphagan, Koenig, y Larson, 2000; Schwab y Petersen, 1990).

Otro de los mecanismos que explicaría el efecto de amortiguamiento de ciertas prácticas religiosas es la integración y el apoyo social que brinda la pertenencia a una comunidad. El apoyo social parece reducir el sentido de amenaza en situaciones difíciles, y promueve estados psicológicos positivos que tienen efectos fisiológicos y psicológicos deseables (Krause, 2002, 2006; Masten y Reed, 2002; Strawbridge et al., 1997; Cohen y Lemay, 2007; Dein y Stygall, 1997; Benjamins, Musick, Gold, y George, 2003; Cohen, 2004; McIntosh, Silver y Wortman, 1993; Mackenzie et al 2000). Al ser la práctica religiosa la que pone en marcha este apoyo social, esta se comportaría como la causa última de estos efectos beneficiosos (Levin, 1996; Thoresen et al., 2001; Watts, 2001).

Finalmente, otros diseños sostienen que determinadas prácticas religiosas como la oración y la meditación ayudan a regular las emociones proporcionando estabilidad emocional (Watts, 2007; McFadden y Levin, 1996).

Ahora bien, podrían señalarse una serie de falencias y limitaciones en estos diseños teóricos. En primer lugar, la teoría del afrontamiento que postulan muchos de los citados estudios adolece de un problema metodológico y de diseño fundamental. Se trata de su incapacidad de descartar la intervención o incidencia de variables externas, desconocidas o no consideradas en el estudio. El propósito mismo de la investigación empírica es descubrir las relaciones que se establecen entre variables, lo cual exige distinguirlas clara y sistemáticamente de otras variables extrañas. Esto hace a la misma validez interna del diseño metodológico (Kazdin, 2001: 16). 
Para poder establecer una relación causal o, al menos una correlación entre prácticas religiosas específicas y la capacidad de afrontamiento, este modelo debería descartar todas aquellas otras variables que podrían incidir en la capacidad de afrontamiento como puede ser las características de la personali$\mathrm{dad}^{5}$, los sucesos en la vida de los participantes, los cambios madurativos y de desarrollo, la incidencia de los métodos de observación o recolección de datos, etc. (Harrison et al., 2001; Pargament, 1997: 285). A su vez, dentro de esta capacidad de afrontamiento deberían discriminarse las experiencias religiosas específicas, las situaciones y las variaciones o formas en las que un individuo o población las traduce en capacidad de afrontamiento. Para ello es necesario determinar (1) el nivel de desarrollo cognitivo y espiritual del individuo, (2) el tipo de problema o factor de estrés, (3) el contexto situacional y cultural específico en el que tiene lugar el afrontamiento, (4) cambios en el proceso de afrontamiento a lo largo del tiempo, (5) el grupo específico o la población que se estudia, y (6) la naturaleza multidimensional de la religión y la espiritualidad (Pargament, Koenig et al., 2000; Koenig, 1995; Pargament, 1997; Chatters, 2000; Ark, Hull, Husaini, y Craun, 2006; Bjorck et al., 1997; Nooney y Woodrum, 2002; Maynard et al., 2001).

Por otro lado, podría discutirse si el apoyo social que proporcionaría la pertenencia a una Iglesia tiene efectos independientes y diferentes al apoyo social que podría brindar la pertenencia a cualquier otra comunidad secular.

Estas falencias citadas son suficientes para ilustrar los vacíos que aún dejan abiertos las fundamentaciones teóricas formuladas por las investigaciones psicoclínicas respecto al potencial psicoterapéutico de las prácticas religiosas. Estas no parecen explicitar suficientemente las propiedades o la naturaleza de las dinámicas específicas que operarían en las prácticas espirituales y religiosas representando una auténtica amenaza de validez del constructo.

Probablemente, si estos estudios recogieran, o al menos consideraran, las explicaciones que las mismas tradiciones religiosas ofrecen respecto al bienestar psíquico y espiritual que proporcionan determinadas prácticas espirituales, las ciencias de la salud mental podrían formular constructos teóricos más comprehensivos y específicos.

${ }^{5}$ Robbins, Francis y Edwards (2008) encontraron que son las características de personalidad de las personas que oran las que pueden explicar gran parte de este efecto. 
De un modo u otro, el tipo de pregunta o abordaje epistémico en la gran mayoría de estos estudios omite en sus postulaciones teóricas el análisis de las propiedades únicas o emergentes del complejo fenómeno que representa la espiritualidad. Es decir, el interés está puesto en dilucidar los mecanismos psicológicos específicos de una técnica, sin importar las propiedades que definen a esa técnica como una práctica espiritual.

Actualmente el modelo de apropiación de prácticas originalmente religiosas que más consenso tiene es aquel que traduce las prácticas espirituales a técnicas de concentración. Su objetivo es medir en términos de eficiencia una práctica religiosa, a modo de un cierto método o técnica psicoterapéutica, sin suscribir en absoluto a las teorías o principios espirituales que los sustentan. (Williams y Swales, 2004). Tales estudios operarían sobre un eclecticismo técnico que no encuentra motivos para indagar en los supuestos antropológicos, religiosos o culturales de las prácticas de procedencia espiritual.

Siguiendo este modelo ecléctico de integración se han llevado a cabo múltiples estudios respecto a la efectividad de técnicas de meditación budistas e hindúes. Es difícil definir la meditación, ya que cada una de estas tradiciones religiosas usa el término de diferentes maneras. Pero en orden a ser ilustrativos podríamos referirnos a la extensa literatura que se esfuerza por definir la atención plena como una tecnología eficiente para manipular la atención, promover la relajación y la autoexploración, independientemente de su contenido religioso, cultural e ideológico original (Langer y Moldoveanu, 2000; Kabat-Zinn, 2003).

La dificultad que supone este modelo de reapropiación de la técnica radica -a nuestro entender- en su mismo proceso de reinvención de la práctica espiritual. Este traza un proceso de redefinición de la práctica espiritual a través del cual se establecen nuevos parámetros de eficiencia. Ahora, estos nuevos parámetros o criterios muchas veces son ajenos al sustrato antropológico y cosmológico en el cual han sido originalmente formulados. De ello resulta la dificultad para poder determinar si los estudios versan o no sobre el potencial psicoterapéutico de una práctica espiritual o si se trata de una nueva técnica cuyos niveles de eficiencia son explicados por las diversas escuelas de la psiquiatría y la psicología moderna. 
En línea con Torneatto y Nguyen (2007), Baer (2003) y Orgiles (2013) podríamos argumentar que no habría soporte empírico alguno que pruebe la utilidad propia y específica del mindfulness. En la medida que la práctica del mindfulness fue reducida a una serie de ejercicios mentales, ella no parece aportar ningún potencial psicoterapéutico original, o al menos distinto a los recursos que en los años cincuenta fueron pautados, sistematizados y medidos por la escuela cognitiva-conductual: la estrategia de la exposición a los propios pensamientos o sensaciones fisiológicas, la habilidad meta-cognitiva de diferenciar los pensamientos perturbadores del propio yo, y las técnicas de autocontrol y relajación ya eran implementadas en la psicoterapia mucho antes que el mindfulness se pusiera de moda. Luego, cabe preguntar, ¿cuál es la estrategia nueva u original que propone el mindfulness o las prácticas de atención plena para la psicoterapia contemporánea? $\mathrm{O}$ se atiende al contexto espiritual del mindfulness o dicha práctica aparentemente no representaría novedad alguna para la psicoterapia.

La meditación budista es un estilo de vida sustentado por un particular tipo de espiritualidad, con un concomitante estilo cognitivo. No es un proceso computacional, o una técnica que pueda ser adquirida mecánicamente. La atención plena, tal como ha sido operacionalizada en la gran mayoría de los estudios psicoclínicos difiere de su uso budista original (Marlatt et al., 2004). Ciertamente, en los estudios clínicos interesa medir los niveles de eficiencia de ciertas técnicas o prácticas. Pero si en dichos estudios se despoja la práctica del mindfulness de su contexto ético y cultural, podrían estar midiendo efectos muy diferentes a los efectos que se siguen originalmente de dicha práctica espiritual. En este sentido, esta tecnificación del mindfulness tiene efectos potencialmente negativos para la misma investigación psicoclínica.

Hoy disponemos de investigaciones que han desafiado este modelo ecléctico de integración.

Se trata de estudios que atienden y seleccionan variables que se explican fundamentalmente en el contexto cosmológico o teológico propio de cada práctica espiritual. En esta línea podemos citar los trabajos que prestan particular atención a las diferencias específicas de una amplia variedad de tipos de meditación, incluidas las técnicas de atención plena, vipassana y budista vajrayana (Andresen, 2000). 
En lugar de retraducir la práctica espiritual a una técnica, estos estudios procuran entenderla y medir variables que se explican en virtud de su propio marco religioso o cosmológico. Atienden al marco teórico que proveen las tradiciones religiosas en cuanto que en ellas pueden discriminarse las diversas variables que afectan al bienestar emocional del paciente. Después de todo, los diferentes tipos de meditación además de un tipo de relajación general producen diferentes cambios específicos o variables a las que la investigación psicoclínica solo puede acceder en la medida que contemple el contexto teórico que las sustenta.

\section{§4. Amenaza de inVAlideZ EXTERnA}

Finalmente, cabe citar la posibilidad de que estas investigaciones sobre espiritualidad y salud adolezcan de lo que se reconoce como la amenaza de invalidez externa. Esta se refiere a la imposibilidad de generalizar los hallazgos de dichas investigaciones sea porque el marco, la variable o la población establecida no puede generalizarse y extenderse a otras variables, poblaciones (Kazdin, 2001: 16).

La oración practicada en una población específica (comunidad de enfermos terminales) remite a resultados específicos que difícilmente pueden generalizarse a poblaciones, escenarios, tiempos, medidas y características que no sean las de esa investigación concreta. Esto nos remite nuevamente al problema de que el análisis del comportamiento de una variable en una población o muestra relativamente grande puede significar y referir un comportamiento absolutamente diverso.

La invalidez externa señala las dificultades y obstáculos que existen para trasladar a la práctica los datos obtenidos en las investigaciones sobre espiritualidad. En líneas generales, hasta diez años atrás los estudios desarrollados sobre la temática que nos ocupa se centraban simplemente en presentar la espiritualidad o las experiencias religiosas como recursos psicológicos cuyo mayor efecto es ayudar a prevenir enfermedades en lugar de curarlas. La evidencia de su efectividad terapéutica era débil y se limitaba a problemas que estaban claramente relacionados con el estrés (Canter, 2003). Aún más, 
muchos de los estudios analizados incluían clientes que no estaban clínicamente afectados y no buscaban tratamiento para algún trastorno o problema particular. Se trataba de estudios, por esto mismo, no representativos clínicamente.

Un problema metodológico central de estos diseños fue su incapacidad por definir bajo qué circunstancias específicas la religión tiene funciones curativas, para qué tipo de paciente, y para cuál tipo de dolencia resulta más beneficiosa. La cuestión a resolver es qué tratamiento, administrado por quién, en qué circunstancia, para qué tipo de paciente y con qué problema resulta el más beneficioso. Preguntas de cuya respuesta depende en definitiva la validez externa.

Desde principios del año 2000 hasta la fecha se han publicado numerosos estudios que apuntan la necesidad de mejorar la calidad de las mediciones empíricas: sea aumentando el rigor de las mediciones, innovando los instrumentos de medición, aumentando el tamaño de las muestras, alargando el seguimiento, o extendiendo el control sobre otras variables que podrían incidir en la muestra etc. Esta tarea de depuración metodológica ha sido implementada en una infinidad de estudios empíricos recientes respecto a la efectividad del mindfulness para tratar los trastornos de ansiedad y de depresión, entre otra amplia variedad de problemas psicológicos.

Pero tales estudios cualitativamente superiores en su abordaje metodológico suponen otro tipo de reducción de carácter epistémico y ontológico. Naturalmente los diseños de la investigación psicoclínica están interesados no en esclarecer las propiedades o las dinámicas per se de la vida espiritual del paciente o de una población, sino en determinar únicamente los posibles efectos psicoterapéuticos de las prácticas religiosas. Dicho en otros términos, busca traducir o transcribir la dinámica espiritual a su concomitante dinámica psíquica.

Este tipo de reduccionismo puede excluir de suyo la posibilidad de admitir, por ejemplo, una nota esencial a la mayoría de las religiones como es la trascendencia. Un estudio de la religión que excluye la consideración de esta variable parece tener límites para comprender su objeto de estudio. Ciertamente, la trascendencia no es una variable directamente observable o susceptible de ser sometida a un diseño experimental. No obstante, en la medida 
que la psicología no considere la posibilidad de que operen en las estructuras psíquicas dinámicas trascendentes a ellas podría estar excluyendo un aspecto crítico y central de un fenómeno que constituye como tal a la espiritualidad (Vergote, 1969: 5-21).

La implementación de un reduccionismo de tipo ontológico impide de suyo una integración genuina de la espiritualidad en un modelo o práctica psicoterapéutica. Pues al negar las notas propias y la relativa autonomía de una dimensión espiritual, se corre el riesgo de que ellas en sí mismas sean subsumidas o reducidas a una práctica clínica o a una serie de ejercicios mentales.

Por otro lado, el reduccionismo ontológico, al no comprender las dinámicas espirituales y psicológicas como realidades o procesos relativamente autónomos e independientes, puede resultar incapaz de reconocer cuadros clínicos donde un paciente pueda tener una vida espiritual rica mientras padece una enfermedad mental o, por el contrario, ser psicológicamente saludable pero sufrir profundos problemas espirituales (Welch, 1996: 98) ${ }^{6}$.

En este sentido, estas modalidades de reduccionismo podrían poseer un valor heurístico considerable en cuanto que permiten formular esquemas comprensivos sencillos susceptibles de ser traducidos a diseños experimen-

\footnotetext{
${ }^{6}$ En este sentido, el desarrollo religioso y espiritual puede estar relacionado con la integridad psicológica pero no necesariamente con la salud mental. El bienestar espiritual puede ser una expresión de salud espiritual o madurez, pero no es idéntico a ellos (cf. Mansager, 2000; Merton, 1991; O'Connor, 2005: 53; von Balthasar, 2000). El fin de las prácticas religiosas no es mantener la adaptación y el funcionamiento social del individuo (Horwtiz, 2002; Horwitz y Wakefield, 2007; Conrad, 2007; Shuman y Meador, 2003; Chamberlain y Hall, 2000: 338; Chatters, 2000). Su finalidad tiene un enfoque relacional y trasciende el bienestar psico-social que procuran garantizar las ciencias de la salud mental (Ai, 2000; Mohan, Mohan, Roy, Basu y Viranjini, 2004). Debido a que la psicología carece de una base teórica o espiritual, la psicología tradicional ha tenido dificultades para incorporar conceptos relacionados con el bienestar espiritual (Charry, 2001).

Desde este punto de vista, los cristianos no son inmunes a las enfermedades mentales y las personas que padecen alguna disfunción o trastorno en su salud mental pueden responder bien a Dios, aunque la unión creciente con Dios generalmente está relacionada con una personalidad más integrada (Welch, 1982: 75-76). Otro ejemplo de esta forma de pensar se puede encontrar en la obra de Merton (1991), quien sostiene que si bien la vida cristiana ascética presupone un nivel normal de madurez psicológica y la ausencia de ansiedad o depresión graves, el objetivo de la vida religiosa es para unir a la persona con Dios en Cristo, no promover un funcionamiento normal como es el caso en la psiquiatría. La vida ascética no ofrece una respuesta a los problemas mentales graves, y de hecho podría empeorarlos debido a las rigurosas exigencias de las prácticas religiosas y la vida comunitaria (cf. Rahner, 1965: 52).
} 
tales -tal como argumentan Corveleyn, 1996; Watts, 2002: 4; Kistler, 2006; Schaffner, 2006; Poirier, 2006-. No obstante, creemos que el costo de estas simplificaciones es particularmente problemático en tanto que facilita comprensiones erróneas de un fenómeno tan complejo como es la religión.

\section{CONSIDERACIONES FINALES}

El reduccionismo es un proceso necesario e ineludible de la investigación empírica. Ahora bien, estos diseños o recortes metodológicos definen un contexto o diseño de investigación que condiciona fuertemente los resultados y el modo en que son interpretados los datos obtenidos. De aquí la necesidad de una instancia de reflexión epistémica capaz de dar cuenta de las distorsiones o límites comprehensivos a los que puedan dar lugar tales reduccionismos.

El mismo carácter fragmentario que posee un diseño experimental exige una instancia de discernimiento meta-teórico que evalúe el impacto de los recortes metodológicos implementados sobre los resultados obtenidos. Esta tarea de análisis es la que permite relativizar la información del diseño como la respuesta a un particular instrumento de selección, observación, medición y análisis pero que en absoluto representa un conocimiento acabado del fenómeno.

Hemos mostrado cómo las diversas modalidades de reduccionismo metodológico atenta contra los mismos diseños de investigación. La traducción del complejo fenómeno de la espiritualidad a una variable ambigua, es decir, mal delimitada e inespecífica atenta contra la misma validez interna del diseño de investigación. La imposibilidad de justificar teóricamente los mecanismos de acción específicos de las concomitantes dinámicas psíquicas de las prácticas espirituales $-y$ la dificultad de diferenciarlos de otras variables extrañas que podrían intervenir- se convierte en una amenaza contra la misma validez del constructo. Finalmente, señalamos la invalidez externa que amenaza a muchas de estas investigaciones en cuanto que en ellas existe dificultad para poder generalizar sus resultados, sea porque no definen el tipo de paciente y la dolencia específica que se beneficiaría de una práctica religiosa, o sea porque no delimitan las circunstancias específicas bajo las cuales la religión o una determinada práctica espiritual puede tener funciones curativas. 
Hemos dado cuenta de una serie de contradicciones y límites que se siguen de la implementación de los reduccionismos metodológicos en la investigación psicoclínica señalando particularmente que aquel no constituye en sí mismo un camino capaz de garantizar la objetividad y la racionalidad científica.

La amenaza de la validez interna, la invalidez del constructo teórico y la invalidez externa de una investigación psicoclínica muchas veces no solo son consecuencias de insuficientes resguardos metodológicos sino justamente resultado de la misma aplicación de los requisitos o reduccionismos metodológicos. Esto nos conduce a una contradicción o absurdo: aquello que debería garantizar el abordaje racional de los fenómenos espirituales y religiosos opera muchas veces como facilitador de comprensiones erróneas e incompletas del fenómeno.

La investigación psicoclínica no puede ser concebida como una disciplina encerrada en sus propios límites. Ella debe complementarse, o aún más propiamente, fundamentarse y hallar justificación en otros dominios conceptuales. Si se pretende hacer justicia, en este caso, al estudio de los efectos psicoterapéuticos de la espiritualidad, este no puede dejar de estar fundamentada sobre tradiciones teológicas y espirituales, a saber, elementos que históricamente han sido excluidos de toda consideración racional o científica. Se trata de una extraña paradoja: en la medida en que la investigación psicoclínica incorpore o atienda a elementos y principios aparentemente ajenos a la racionalidad científica, las demostraciones científicas podrían adquirir mayor fuerza explicativa.

\section{REFERENCIAS BIBLIOGRÁFICAS}

Aaron, K. F., Levine, D., Burstin, H. R. (2003). African American church participation and health care practices. Journal of General International Medicine (18), 908-913.

Ai, A., Dunkle, R., Peterson, C., Bowlling, S. (1998). The role of private prayer in psychological recovery among midlife and aged patients following cardiac surgery. The Gerontological Society of America (38), 591-601. 
American Psychological Association. Presidential Task Force on Evidence-Based Practice. (2006). Evidence-based practice in psychology. American Psychologist (61), 271-285.

American Psychological Association (2010). APA Dictionary of Clinical Psychology. Washington, D.C.: American Psychological Association.

Anderson, C., Kim, C. (2003). Evaluating Treatment Efficacy with Single-Case Designs. En M. Roberts, \& S. Illardi (eds.), Handbook of Research Methods in Clinical Psychology (pp. 73-91). Oxford, Inglaterra: Blackwell.

Andler, D. (2006). Federalism in science-complementarity vs. perspectivism: Reply to Harré. Synthese (151), 519-522.

Arcury, T. A., Quandt, S. A., McDonald, J., Bell, R. A. (2000). Faith and health self-management of rural older adults. Journal of Cross-Cultural Gerontology (15), 55-74.

Ark, P. D., Hull, P. C., Husaini, B. A., Craun, C. (2006). Religiosity, religious coping styles, and health service use. Journal of Gerontological Nursing, 32(8), 20-29.

Baetz, M., Griffin, R., Bowen, R., Koenig, H., Marcoux, E. (2004). The association between spiritual and religious involvement and depressive symptoms in a Canadian population. Journal of Nervous and Mental Disease (192), 818-822.

Baker, T. B., McFall, R. M., Shoham, V. (2008). Current status and future prospects of clinical psychology: Toward a scientifically principled approach to mental and behavioral health care. Psychological Science in the Public Interest, 9(2), 67-103.

Balboni, T. A., Fitchett, G., Handzo, G. F., Johnson, K. S., Koenig, H. G., Pargament, K. I., ... Steinhauser, K. E. (2017). State of the science of spirituality and palliative care research Part II: screening, assessment, and interventions. Journal of pain and symptom management, 54(3), 441-453.

Banziger, S., van Uden, M., Janssen, J. (2008). Praying and coping: The relation between varieties of praying and religious coping styles. Mental Health, Religion \& Culture (11), 101-118. 
Barbour, I. (1997). Religion and science: Historical and contemporary issues. San Francisco, CA: Harper San Francisco, 230.

Barlow, D. (1981). On the Relation of Clinical Research to Clinical Practice: Current Issues, New Directions. Journal of Consulting and Clinical Psychology, 49(2), 147-155.

Barrett, L., Dunbar, R., Lycett, J. (2002). Human evolutionary psychology. Princeton, NJ: Princeton University Press.

Benjamins, M. R., Musick, M. A., Gold, D. T., George, L. K. (2003). Age-related declines in activity level: The relationship between chronic illness and religious activities. The Journals of Gerontology, 58B(6), S377-S385.

Bennett, M. R., Hacker, P. M. S. (2003). Philosophical foundations of neuroscience. Malden, MA: Blackwell.

Bjorck, J. P., Lee, Y. S., Cohen, L. (1997). Control beliefs and faith as stress moderators for Korean American versus Caucasian American protestants. American Journal of Community Psychology (25), 61-72.

Bosworth, H. B., Park, K., McQuoid, D. R., Hays, J. C., Steffens, D. C. (2003). The impact of religious practice and religious coping on geriatric depression. International Journal of Geriatric Psychiatry (18), 905-914.

Brown, L. (1994). The human side of prayer: The psychology of praying. Birmingham, AL: Religious Education Press.

Byrd, R. (2001). Positive therapeutic effects of intercessory prayer in a coronary care unit population. In L. Francis, J. Astley (eds.), Psychological perspectives on prayer (pp. 156-163). Leominster, UK: Gracewing.

Canter, P. H. (2003). The therapeutic effects of meditation. British Medical Journal (326), 1049-1050.

Chatters, L. M. (2000). Religion and health: Public health research and practices. Annual Review of Public Health (21), 335-367.

Cohen, S. (2004). Social relationships and health. American Psychologist (59), 676-684.

Cohen, S., Lemay, E. (2007). Why would social networks be linked to affect and health practices? Health Psychology (26), 410-417. 
Contrada, R. J., Goyal, T. M., Cather, C., Rafalson, L., Idler, E. L., Krause, T. J. (2004). Psycho-social factors in outcomes of heart surgery: The impact of religious involvement and depres-sive symptoms. Health Psychology (23), 227-238.

Cooper, T. D. (2007). Dimensions of evil: Contemporary perspectives. Minneapolis, MN: Fortress, 30-38.

Davidson, R. J., Pizzagalli, D., Nitschke, J., Putnam, K. (2002). Depression: Perspectives from affective neuroscience. Annual Review of Psychology (53), 545-574.

Davies, P. (1998). Teleology without: Purpose through. En R. Russell, W. Stoeger, F. Ayala (eds.), Evolutionary and molecular biology: Scientific perspectives on divine action (pp. 151-162). Vatican City State: Vatican Observatory.

Dein, S., Stygall, J. (1997). Does being religious help or hinder coping with chronic illness? A critical literature review. Palliative Medicine (11), 291298.

Dillon, M., Wink, P. (2003). Religiousness and spirituality: Trajectories and vital involvement in late adulthood. En M. Dillon (ed.), Handbook of the sociology of religion (pp. 179-189). Cambridge: Cambridge University Press.

Dillon, M., Wink, P. (2007). In the course of a lifetime: Tracing religious belief, practice, and change. Berkeley, CA: University of California Press.

Dossey, L. (1997). Healing words: The power of prayer and the practice of medicine. New York: Harper.

Ellis, G. (2002). Intimations of transcendences: Relations in the mind and God. En R. Russell, N. Murphy, T. Meyering, M. Arbib (eds.), Neuroscience and the person: Scientific perspectives on divine action (pp. 449474). Vatican City State: Vatican Observatory.

Ellison, C. G., Levin, J. S. (1998). The religion-health connection: Evidence, theory, and future directions. Health Education and Behavior (25), 700720.

Faucher, L. (2006). What's behind a smile? The return of mechanism: Reply to Schaffner. Synthese (151), 403-409. 
Finger, M., Rand, K. (2003). Addressing Validity Concerns in Clinical Psychology Research. En M. Roberts, S. Illardi (eds.), Handbook of Research Methods in Clinical Psychology (pp. 13-30). Oxford, Inglaterra: Blackwell.

Finney, J., Malony, H. (2001). An empirical study of contemplative prayer as an adjunct to psychotherapy. En L. Francis, J. Astley (eds.), Psychological perspectives on prayer (pp. 359-367). Leominster, UK: Gracewing.

Fitchett, G. Risk, J. L. (2009). Screening for spiritual struggle (4-1-12). J Pastoral Care Counsel (63).

Fitchett, G., Rybarczyk, B. D., DeMarco, G. A., Nicholas, J. J. (1999). The role of religion in medical rehabilitation outcomes: a longitudinal study. Rehabil Psychol (44), 333-353.

Flannelly, K. J., Koenig, H. G., Ellison, C. G., Galek, K., Krause, N. (2006). Belief in life after death and mental health findings from a national survey. The Journal of Nervous and Mental Disease (194), 524-529.

Francis, L., Evans, T. (2001). The psychology of Christian prayer: A review of empirical research. En L. Francis, J. Astley (eds.), Psychological perspectives on prayer (pp. 2-22). Leominster, UK: Gracewing.

Gallego, S., de los Bueis, F., Prats, S., Ortega-Verdaguer, A., Antonín, P., Serra, M., Rossy, C., Revuelto, A., Ciudad, E., Álvarez-Segura, M. (2016). Contribuciones y Confusiones de la Espiritualidad en la salud mental. Gaceta de Psiquiatría Universitaria, 4(12), 353-358.

George, L. K., Ellison, C. G., Larson, D. B. (2002). Explaining the relationships between religious involvement and health. Psychological Inquiry (13), 190-200.

Harris, W., Gowda, M., Kolb, J., Strychacz, C., Vacek, J. et al. (2001). A randomized, controlled trail of the effects of remote, intercessory prayer on outcomes in patients admitted to the coronary care unit. En L. Francis, J. Astley (eds.), Psychological perspectives on prayer (pp. 164-176). Leominster, UK: Gracewing.

Harrison, M. O., Koenig, H. G., Hays, J. C., Eme-Akwari, A. G., Pargament, K. I. (2001). The epidemiology of religious coping: A review of recent literature. International Review of Psychiatry (13), 86-93. 
Horwtiz, A. (2002). Creating mental illness. Chicago: University of Chicago. Hummer, R. A., Rogers, R. G., Nam, C. B., Ellison, C. G. (1999). Religious involvement and U.S. adult mortality. Demography (36), 273-285.

Idler, E. L. (1995). Religion, health, and nonphysical senses of self. Social Forces (74), 683-704.

Idler, E. L., Kasl, S. V. (1997a). Religion among disabled and nondisabled persons I: Cross-sectional patterns in health practices, social activities, and well-being. Journal of Gerontology: Social Sciences, 52B(6), S294-S305.

Idler, E. L., Kasl, S. V. (1997b). Religion among disabled and nondisabled persons II: Attendance at religious services as a predictor of the course of disability. Journal of Gerontology: Social Sciences, 52B(6), S306-S316.

Kendler, K. S., Gardner, C. O., Prescott, C. A. (1997). Religion, psychopathology, and substance use and abuse: A multimeasure, genetic-epidemiological study. The American Journal of Psychiatry (154), 322-329.

Kennedy, P., Drebing, C. E. (2002). Abuse and religious experience: A study of religiously com-mitted evangelical adults. Mental Health, Religion \& Culture (5), 225-237.

Kazdin, A. (2001). Métodos de investigación en psicología clínica. México, D.F.: Prentice Hall.

Kim, J. (2006). Emergence: Core ideas and issues. Synthese (151), 547-559.

Kim, D., Moon, Y., Kim, H., Jung, J., Park, H., Suh, H. et al. (2005). Effect of Zen meditation on serum nitric oxide activity and lipid peroxidation. Progress in Neuro-Psychopharmacology and Biological Psychiatry (29), 327-331.

Kirby, S. E., Coleman, P. G., Daley, D. (2004). Spirituality and well-being in frail and nonfrail older adults. Journal of Gerontology: Psychological Sciences, 59B(3), P123-P129.

Kistler, M. (2006). New perspectives on reduction and emergence in physics, biology and psychology. Synthese (151), 311-312.

Koenig, H. G. (1995). Religion as cognitive schema. The International Journal for the Psychology of Religion (5), 31-37. 
Koenig, H. G. (2001). Religion and medicine IV: Religion, physical health, and clinical implications. International Journal of Psychiatry in Medicine (31), 321-336.

Koenig, H. G., Cohen, H. J., Blazer, D. G., Pieper, C., Meador, K. G., Shelp, F. et al. (1992). Religious coping and depression among elderly, hospitalized medically ill men. The American Journal of Psychiatry (149), 1693-1700.

Koenig, H. G., Hays, J. C., Larson, D. B., George, L. K., Cohen, H. J., McCullough, M. E. et al. (1999). Does religious attendance prolong survival? A six-year follow-up study of 3,968 older adults. Journal of Gerontology: Medical Sciences, 54A(7), M370-M376.

Krause, N. (2002). Church-based social support and health in old age: Exploring variations by race. Journal of Gerontology: Social Sciences, 57B(6), S332-S347.

Krause, N. (2006). Church-based social support and mortality. Journal of Gerontology: Social Sciences, 61B(3), S140-S146.

Krause, N., Tran, T. V. (1989). Stress and religious involvement among older Blacks. Journal of Gerontology: Social Sciences, 44(1), S4-S13.

Krause, M., Chatters, L., Meltzer, T., Morgan, D. (2000). Using focus groups to explore the nature of prayer in late life. Journal of Aging Studies (14), 191-212.

Krause, N., Liang, J., Shaw, B. A., Sugisawa, H., Kim, H., Sugihara, Y. (2002). Religion, death of a loved one, and hypertension among older adults in Japan. Journal of Gerontology: Social Sciences, 57B(2), S96-S107.

Laird, S. P., Snyder, C. R., Rapoff, M. A., Green, S. (2004). Measuring private prayer: Devel-opment, validation, and clinical application of the multidimensional prayer inventory. The International Journal for the Psychology of Religion (14), 251-272.

Levin, J. S. (1996). How religion influences morbidity and health: Reflections on natural history, salutogenesis, and host resistance. Social Science \& Medicine (43), 849-864.

Levin, J. S. (2001). God, faith, and health: Exploring the spirituality-healing connection. New York: John Wiley \& Sons. 
Levin, J. S., Chatters, L. M. (1998). Religion, health, and psychological well-being in older adults: Findings from three national surveys. Journal of Aging and Health (10), 504-531.

Levin, J. S., Chatters, L., Taylor, R. (2005). Religion, health and medicine in African Americans: Implications for physicians. Journal of the National Medical Association (97), 237-249.

MacIntyre, A. (1992). How psychology makes itself true- or false. In S. Koch, D. E. Leary (eds.), A century of psychology as science (pp. 897-920). Washington DC: American Psychological Association.

Mackenzie, E. R., Rajagopal, D. E., Meilbohm, M., Lavizzo-Mourey, R. (2000). Spiritual support and psychological well-being: Older adults' perceptions of the religion and health connection. Alternative Therapies in Health and Medicine, 6(6), 37-45.

Mako, C., Galek, K., Poppito, S. R. (2006). Spiritual pain among patients with advanced cancer in palliative care. J Palliat Med. (9), 1106-1113.

Marras, A. (2006). Emergence and reduction: Reply to Kim. Synthese (151), 561-569.

Maselko, J., Kubzansky, L., Kawachi, I., Staudenmayer, J., Berkman, L. (2006). Religious service attendance and decline in pulmonary function in a high-functioning elderly cohort. Annals of Behavioral Medicine (32), 245-253.

Masters, K. S. (2005). Research on the healing power of distant intercessory prayer: Disconnect between science and faith. Journal of Psychology and Theology (33), 268-277.

Masten, A. S., Reed, M.-G. J. (2002). Resilience in development. In C. R Snyder, S. J. Lopez (eds.), Handbook of positive psychology (pp. 74-88). Oxford: Oxford University Press.

Maynard, E. A., Gorsuch, R. L., Bjorck, J. P. (2001). Religious coping style, concept of God, and personal religious variables in threat, loss, and challenge situations. Journal for the Scientific Study of Religion (40), 65-74.

McCullough, M. E., Larson, D. B. (1999). Religion and depression: A review of the literature. Twin Research (2), 126-136. 
McCullough, M. E., Pargament, K., Thoresen, C. (2000). The psychology of forgiveness: History, conceptual issues, and overview. En M. McCullough, K. Pargament, C. Thoresen (eds.), Forgiveness: Theory, research and practice (pp. 1-14). New York: Guilford Press.

McCullough, M. E., Hoyt, W. T., Larson, D. B., Koenig, H. G., Thoresen, C. (2000). Religious involvement and mortality: A meta-analytic review. Health Psychology (19), 211-222.

McCullough, M. E. (2001). Religious involvement and mortality: Answers and more questions. En T. Plante, A. Sherman (eds.), Faith and health: Psychological perspectives (pp. 53-74). New York: Guilford Press.

McFadden, S. H., Levin, J. S. (1996). Religion, emotions, and health. En C. Magai, S. H. McFadden (eds.), Handbook of emotion, adult development, and aging (pp. 349-365). San Diego, CA: Academic Press.

McIntosh, D. N., Silver, R. C., Wortman, C. B. (1993). Religion's role in adjustment to a negative life event: Coping with the loss of a child. Journal of Personality and Social Psychology (65), 812-821.

Miller, R. (2001). The positive effect of prayer on plants. En L. Francis, J. Astley (eds.), Psychological perspectives on prayer (pp. 153-155). Leominster, UK: Gracewing.

Mueller, P. S., Plevak, D. J., Rummans, T. A. (2001). Religious involvement, spirituality, and medicine: Implications for clinical practice. Mayo Clinic Proceedings (76).

Murphy, N. (1998). Supervenience and nonreducibility of ethics to biology. En R. Russell, W. Stoeger, F. Ayala (eds.), Evolutionary and molecular biology: Scientific perspectives ondivine action (pp. 463-490). Vatican City State: Vatican Observatory.

Murphy, N. (2005). Philosophical resources for integration. En A. Dueck, C. Lee (eds.), Why psychology needs theology: A radical reformation perspective (pp. 3-27). Grand Rapids, MI: Eerdmans.

Musick, M. A., Traphagan, J. W., Koenig, H. G., Larson, D. B. (2000). Spirituality in physical health and aging. Journal of Adult Development (7), 73-86. 
Nooney, J., Woodrum, E. (2002). Religious coping and church-based social support as predictors of mental health outcomes: Testing a conceptual model. Journal for the Scientific Studyof Religion (41), 359-368.

Oxman, T. E., Freeman, Jr., D. H., Manheimer, E. D. (1995). Lack of social participation or religious strength and comfort as risk factors for death after cardiac surgery in the elderly. Psychosomatic Medicine (57), 5-15.

Pargament, K. I. (1997). The psychology of religion and coping: Theory, research and practice (New ed.). New York: Guilford.

Pargament, K. I. (2002). The bitter and the sweet: An evaluation of the costs and benefits of religiousness. Psychological Inquiry (13), 168-181.

Pargament, K. I., Koenig, H. G., Pérez, L. M. (2000). The many methods of religious coping: Development and initial validation of the RCOPE. Journal of Clinical Psychology (56), 519-543.

Peacocke, A. (1993). Theology for a scientific age: Being and becoming-natural, divine, andhuman. Minneapolis, MN: Fortress Press.

Peacocke, A. (2002). A sound of sheer silence: how does God communicate with humanity. En R. Russell, N. Murphy, T. Meyering, M. Arbib (eds.), Neuroscience and the person: Scientific perspectives on divine action (pp. 215-248). Vatican City State: Vatican Observatory.

Peltzer, K. (2004). Preventive health behavior, personality and religiosity among Black and White South Africans. Studia Psychologica (46), 37-48.

Poirier, P. (2006). Finding a place for elimination in inter-level reductionist activities: Reply to Wimsatt. Synthese (151), 477-483.

Roberts, M., Ilardi, S. (2003). Research Methodology and Clinical Psychology: An Overview. En M. Roberts, S. Ilardi (eds.), Handbook of Research Methods in Clinical Psychology (pp. 3- 12). Oxford, Inglaterra: Blackwell.

Robbins, M., Francis, L., Edwards, B. (2008). Prayer, personality and happiness: A study among undergraduate students in Wales. Mental Health, Religion \& Culture (11), 93-99.

Rumbold B. D. (2003). Caring for the spirit: lessons from working with the dying. Med J Aust, 179(6 Suppl): S11. 
Schaffner, K. F. (2006). Reduction: The Cheshire cat problem and a return to roots. Synthese (151), 377-402.

Schwab, R., Petersen, K. U. (1990). Religiousness: Its relation to loneliness, neuroticism and subjective well-being. Journal for the Scientific Study of Religion (29), 335-345.

Shahabi, L., Powell, L. H., Musick, M. A., Pargament, K. I., Thoresen, C. E., Williams, D. et al. (2002). Correlates of self-perceptions of spirituality in American adults. Annals of Behavioral Medicine (24), 59-68.

Sinclair, S., Pereira, J., Raffin, S. (2006). A thematic review of the spirituality literature within palliative care. $J$ Palliat Med (consultado: 5 de diciembre de 2014), 9(2): 467. Disponible en: <http://www.ncbi.nlm.nih.gov/ pubmed/16629575 1>.

Slife, B. D., Hopkins, R. O. (2005). Alternative assumptions for neuroscience: Formulating a true monism. In B. D. Slife, J. S. Reber, F. C. Richardson (eds.), Critical thinking about psychology: Hidden assumptions and plausible alternatives (pp. 121-147). Washington, DC: American Psychological Association.

Smith, H. (2000). Methodology, comparisons, and truth. En K. Patton, B. Ray (eds.), A magicstill dwells: Comparative religion in the postmodern age (pp. 172-181). Berkeley, CA: University of California.

Steinhauser, K. E., Clipp, E. C., Bosworth, H. B. et al. (2004). Measuring quality of life at the end of life: validation of the QUAL-E. Palliat Support Care (2), 3-14.

Steinhauser, K. E., Voils, C. I., Clipp, E. C., Bosworth, H. B., Christakis, N. A., Tulsky, J.A. (2006). "Are you at peace?": one item to probe spiritual concerns at the end of life. Arch Intern Med (166), 101-105.

Steffen, P. R., Hinderliter, A. L., Blumenthal, J. A., Sherwood, A. (2001). Religious coping, ethnicity, and ambulatory blood pressure. Psychosomatic Medicine (63), 523-530.

Strawbridge, W. J., Cohen, R. D., Shema, S. J., Kaplan, G. A. (1997). Frequent attendance at religious services and mortality over 28 years. American Journal of Public Health (87), 957-961. 
Task Force on Promotion and Dissemination of Psychological Procedures. (1995).Training in and dissemination of empirically-validated psychological treatment: Report and recommendations. The Clinical Psychologist (48), 2-23.

Taylor, C. (2007). A secular age. Cambridge, MA: Belknap, 679 y 704-707.

Thoresen, C. E., Harris, A. H. S., Oman, D. (2001). Spirituality, religion, and health: Evidence, issues, and concerns. En T. Plante, A. Sherman (eds.), Faith and health: Psychological perspectives (pp. 15-52). New York: Guilford.

Vachon, M., Fillion, L., Achille, M., (2009) A conceptual analysis of spirituality at the end of life. Journal of Palliative Medicine, 12(1), 53-59.

Vande Kemp, H. (2000). Gordon Allport's pre-1950 writings on religion: The archival record. En J. A. Belzen (ed.), Aspects in contexts: Studies in the history of psychology of religion (pp. 129-172). Amsterdam: Rodopi.

Van Ness, P. H., Kasl, S. V. (2003). Religion and cognitive dysfunction in an elderly cohort. The Journals of Gerontology. Series B, Psychological Sciences and Social Sciences, 58(1), S21-S29.

Watts, F. (2001). Prayer and psychology. In F. Watts (ed.), Perspectives on prayer (pp. 39-52). London: SPCK.

Watts, F. (2002). Theology and psychology. Aldershot, UK: Ashgate.

Watts, F. (2007). Emotion regulation and religion. En J. Gross (ed.), Handbook of emotion regulation (pp. 504-520). New York: Guilford Press.

WHOQOL Group (1998). Development of the world health organization WHOQOL-BREF quality of life assessment. Psychol Med., 28(3), 551-8

Wink, P. (2003). Dwelling and seeking in late adulthood: The psychosocial implications of two types of religious orientations. Journal of Religious Gerontology, 14(2/3), 101-117.

Zizioulas, J. (2006). Communion and otherness: Further studies in personhood and the church (P. McPartlan, Ed.). London: T \& T Clark. 